\title{
Quantitative Chemical Mapping of Soft-Hard Interfaces on Gold Nanorods
}

\author{
Blanka Janicek ${ }^{1}$, Joshua Hinman ${ }^{2}$, Jordan Hinman ${ }^{2}$, Huei-Huei Chang ${ }^{2}$, Kenneth Suslick ${ }^{2}$, Catherine \\ Murphy $^{2}$, and Pinshane Huang ${ }^{1}$ \\ ${ }^{1}$ Department of Materials Science and Engineering, University of Illinois Urbana-Champaign, Urbana, \\ IL, United States 61801 \\ ${ }^{2}$ Department of Chemistry, University of Illinois Urbana-Champaign, Urbana, IL, United States 61801
}

Soft-hard interfaces at the surface of nanoparticles determine interaction potentials, including the mechanisms of growth, spatial reactivity, colloidal stability, and nanoparticle functionality [1]. For example, soft molecular ligands are thought to guide growth and symmetry breaking in anisotropic nanoparticles. These ligands can also act as soft templates for site-selective deposition of functional coatings [2-4]. Quantitative details regarding the local attachment, distribution, and structure of these softhard interfaces would enable the development of methods for high-yield, monodisperse nanoparticle synthesis for applications ranging from catalysis [5] to drug delivery [6].

Conventional techniques to characterize soft-hard interfaces - such as nuclear magnetic resonance, small angle x-ray scattering, and other bulk methods - lack the spatial resolution necessary to probe key details of the soft-hard interface, including how the surface structure and chemistry varies within and between individual particles [1]. Such limitations are critical in studying nanoparticles, where polydispersity is a defining characteristic and where surface energies and interactions vary widely based on local facet, curvature, and composition. While electron microscopy (EM) can in principle address this challenge, an ideal EM approach requires a combination of: low-background substrates, the ability to quantify elemental distributions of small molecules, and the speed and efficiency necessary to probe multiple nanoparticles. Here we report a particle-by-particle analysis of soft-hard interfaces on gold nanorods (AuNRs) using aberration-corrected scanning transmission electron microscopy (STEM) and electron energy loss spectroscopy (EELS) spectrum imaging on graphene substrates.

In order to demonstrate the ability of EM methods to probe soft-hard interfaces, we investigate anisotropic mesoporous silica functionalization of AuNRs. Mesoporous silica can be deposited on AuNRs with site selectivity to either the ends or the sides. Such growth is thought to be templated by the anisotropic distribution of capping ligand cetyl trimethylammonium bromide (CTAB) [2,3]. We spray deposited these mesoporous silica-coated AuNRs onto suspended graphene substrates. Using EELS spectral imaging, we map the presence of carbon, silicon, and oxygen. We are able to directly observe a mesoporous silica frame with carbon containing pores. On end silica-coated AuNRs, we identify the radial orientation of the pores and a $2.5 \mathrm{~nm}$ average pore size (Figure 1). The side coated AuNR pores are predominantly oriented parallel to the transverse axis with a $1.6 \mathrm{~nm}$ pore size (Figure 2). Furthermore, we observe an increased carbon signal on the surface of the nanoparticles, indicating the presence of a residual CTAB shell directly surrounding the particle (Figure 1d, 2d). Using graphene as a reference for the carbon inelastic scattering cross section, we are able to quantify the CTAB present before and after silica deposition. These results indicate that before deposition, AuNRs are coated with a few-nm thick ligand layer with an average grafting density of 2.1 ligands $/ \mathrm{nm}^{2}$. This density, which corresponds to $\sim 5,000$ ligands/particle, is small when compared to the $\sim 60,000$ ligands/particle on silica-coated AuNRs. Our methods thus represent the first direct, quantitative chemical analysis of soft-hard interfaces of metal nanoparticles. 


\section{References:}

[1] A Smith, et al., Analyst 142 (2017), p. 11.

[2] J Hinman, et al., J. Am. Chem. Soc. 139 (2017), p. 9851.

[3] F Wang et al., Angew. Chemie - Int. Ed. 52 (2013), p. 10344.

[4] S Wu, C Mou, and H Lin, Chem. Soc. Rev. 42 (2013), p. 3862.

[5] R Narayanan and M El-Sayed, Nano Lett. 4 (2004), p. 1343.

[6] E Dreaden, et al., Ther. Deliv. 3 (2012), p. 457.

[7] This work was supported by the Packard Foundation, NSF-MRSEC under Award Number DMR1720633, and NSF grant 1608743. The experimental work was carried out in part in the Frederick Seitz Materials Research Laboratory Central Facilities and made use of the Cornell Center for Materials Research Shared Facilities, which are supported through the NSF MRSEC program (DMR-1719875).
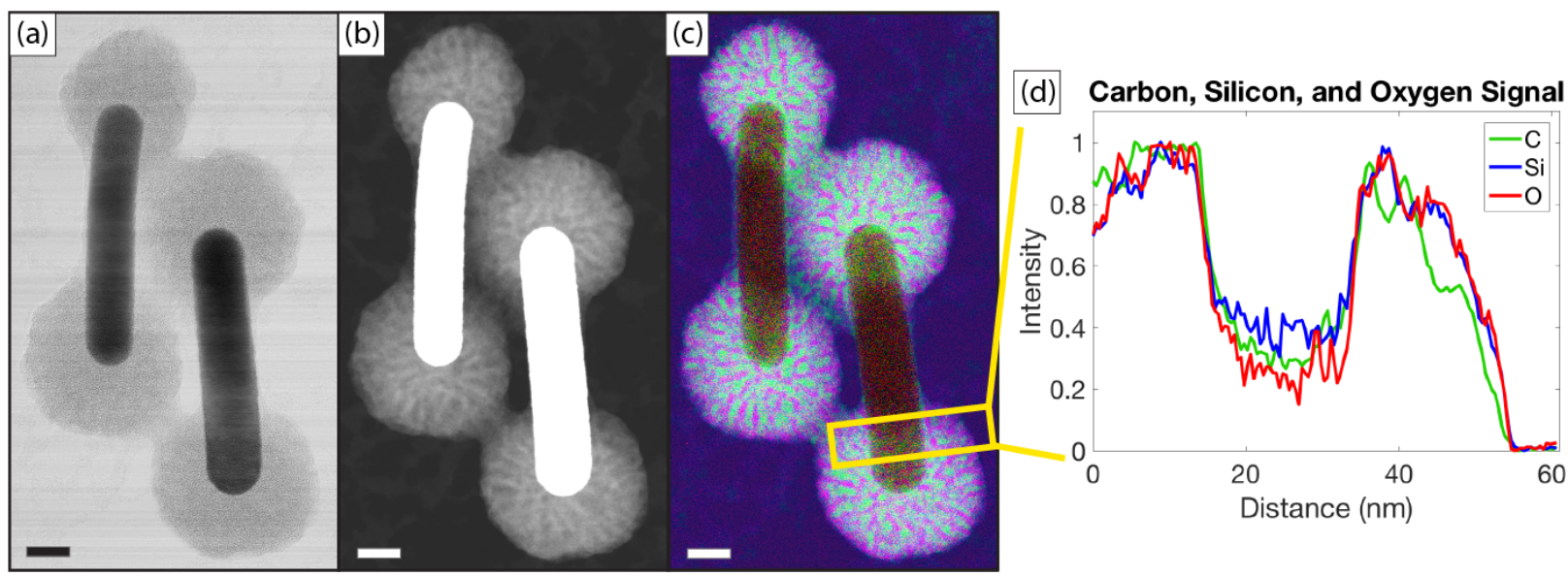

Figure 1. STEM images and color EELS spectrum images of end mesoporous silica-coated AuNRs. All scale bars are $15 \mathrm{~nm}$. C is represented in green, Si in blue, and $\mathrm{O}$ in red. (a) Bright field (BF) and (b) high angle annular dark field (HAADF) STEM images. (c) The EELS compositional map shows silica frame with carbon containing pores. The pore distribution is radial with a $2.5 \mathrm{~nm}$ average size. (d) Line profile of the region indicated with the inset box of the $\mathrm{C}, \mathrm{Si}$, and $\mathrm{O}$ signals. The $\mathrm{C}$ signal opposes the $\mathrm{Si}$ and $\mathrm{O}$, and peaks on the surface of the particle.

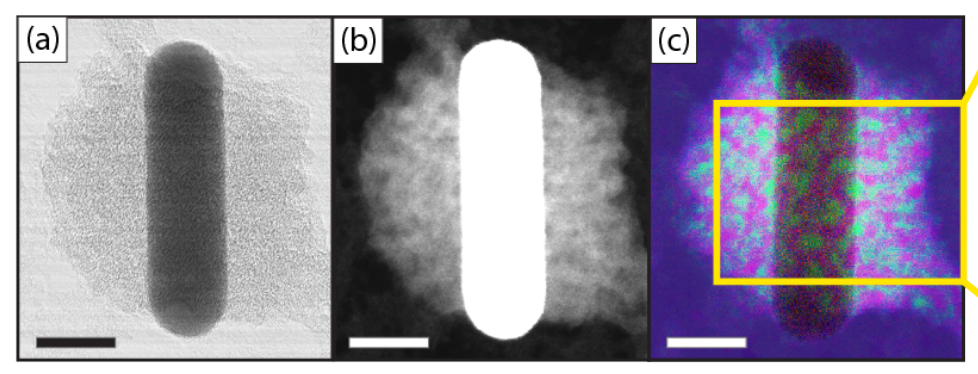

(d) Carbon, Silicon, and Oxygen Signal

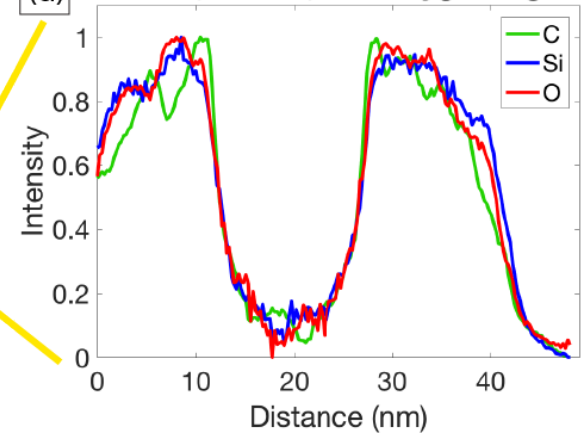

Figure 2. STEM images and color EELS spectrum images of side mesoporous silica-coated AuNRs. All scale bars are $15 \mathrm{~nm}$. $\mathrm{C}$ is represented in green, $\mathrm{Si}$ in blue, and $\mathrm{O}$ in red. (a) BF and (b) HAADF STEM images. c) The EELS compositional map shows the pore distribution is parallel to the transverse axis with a $1.6 \mathrm{~nm}$ pore size. (d) Line profile of the $\mathrm{C}, \mathrm{Si}$, and $\mathrm{O}$ signals where the $\mathrm{C}$ signal opposes $\mathrm{Si}$ and $\mathrm{O}$, and peaks on the surface of the particle. 